\title{
Selective Gene Expression Analysis of Muscular and Vascular Components in Hearts Using Laser Microdissection Method
}

\author{
Ayami Ikeda, ${ }^{1}$ Hisashi Kai, ${ }^{1}$ Hidemi Kajimoto, ${ }^{2}$ Suguru Yasuoka, ${ }^{1}$ \\ Masayoshi Kage, ${ }^{3}$ and Tsutomu Imaizumi ${ }^{1}$ \\ ${ }^{1}$ Division of Cardio-Vascular Medicine, Department of Internal Medicine, Kurume University School of Medicine, \\ 67 Asahimachi, Kurume, Fukuoka 830-0011, Japan \\ ${ }^{2}$ Cardiovascular Research Institute, Kurume University, Kurume 830-0011, Japan \\ ${ }^{3}$ Department of Diagnostic Pathology, Kurume University Hospital, Kurume 830-0011, Japan \\ Correspondence should be addressed to Hisashi Kai, naikai@med.kurume-u.ac.jp
}

Received 22 March 2012; Revised 11 April 2012; Accepted 29 April 2012

Academic Editor: Rei Shibata

Copyright (๑) 2012 Ayami Ikeda et al. This is an open access article distributed under the Creative Commons Attribution License, which permits unrestricted use, distribution, and reproduction in any medium, provided the original work is properly cited.

\begin{abstract}
Background. The heart consists of various kinds of cell components. However, it has not been feasible to separately analyze the gene expression of individual components. The laser microdissection (LMD) method, a new technology to collect target cells from the microscopic regions, has been used for malignancies. We sought to establish a method to selectively collect the muscular and vascular regions from the heart sections and to compare the marker gene expressions with this method. Methods and Results. Frozen left ventricle sections were obtained from Wistar-Kyoto rats (WKY) and stroke-prone spontaneously hypertensive rats (SHR-SP) at 24 weeks of age. Using the LMD method, the muscular and vascular regions were selectively collected under microscopic guidance. Real-time RT-PCR analysis showed that brain-type natriuretic peptide (BNP), a marker of cardiac myocytes, was expressed in the muscular samples, but not in the vascular samples, whereas $\alpha$-smooth muscle actin, a marker of smooth muscle cells, was detected only in the vascular samples. Moreover, SHR-SP had significantly greater BNP upregulation than WKY $(P<0.05)$ in the muscular samples. Conclusions. The LMD method enabled us to separately collect the muscular and vascular samples from myocardial sections and to selectively evaluate mRNA expressions of the individual tissue component.
\end{abstract}

\section{Introduction}

The heart consists of the cardiac muscle, vasculature, and to a lesser extent interstitial infiltrating cells. It is considered that the gene expressions are separately regulated in these tissue components under physiological and diseased conditions $[1,2]$. Although the mRNA expression analysis has been established in the whole myocardium, it is difficult to investigate the expression level of each tissue component.

Recently, the laser microdissection (LMD) method has been developed to isolate specific microscopic regions from tissue samples and separately collect the specimens of interest, which enables us to selectively evaluate the mRNA expression levels in targeted cell clusters in the tissues, especially in malignant tissues [3]. The regions of interest are marked on the monitor of a vertical microscope and cut out by the laser beam under computer control. The isolated samples fall down into collecting tubes, which are subjected to quantitative real-time reverse-transcribed polymerase chain reaction (RT-PCR) or gene-chip/microarray assay. However, the LMD method has not been applied to the heart. Thus, we sought to establish a method to selectively collect the muscular region and arterial region in myocardial sections using the LMD method. The mRNA expression levels of maker genes specific for cardiac myocytes or vascular smooth muscle cells (VSMCs) were analyzed in the muscular and vascular samples, respectively, obtained from rat heart sections.

\section{Methods}

The study protocol was reviewed and approved by the Animal Care and Treatment Committee of Kurume University. 


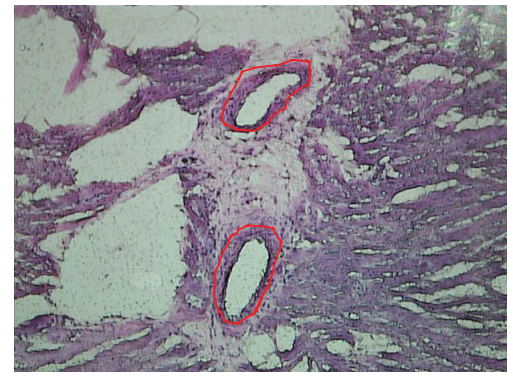

(a)

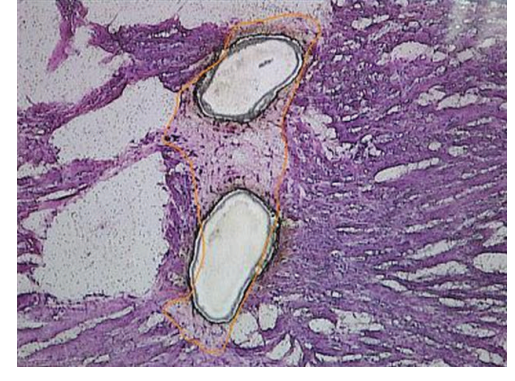

(b)

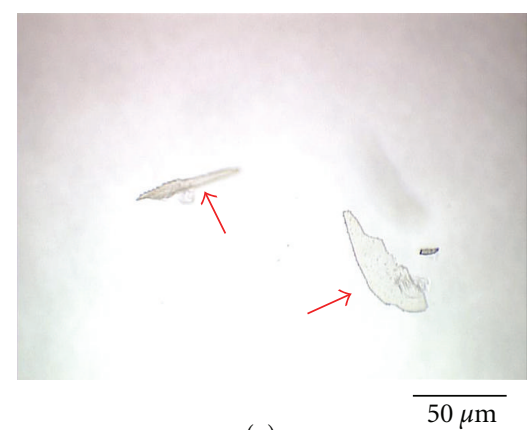

(c)

(A)

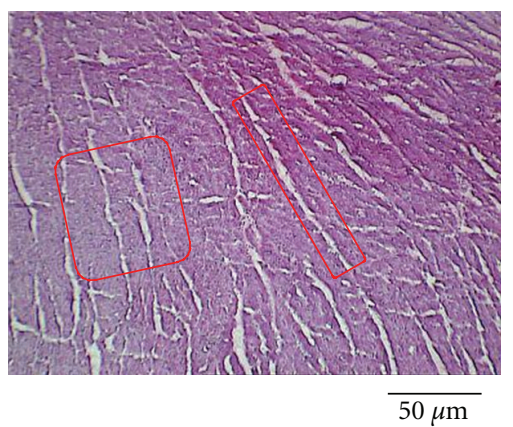

(B)

FIGURE 1: (A) Representative microphotographs demonstrating the sampling of vascular area of the myocardial section obtained from SHRSP using LMD method. (a) Laser cut lines (red lines) were placed on the outside border of the medial smooth muscle layer. (b) Myocardial section after vascular samples were cut off by laser dissection shown. (c) After laser dissection, isolated vascular fragments (red arrows) fell down into the caps of Eppendorf tubes containing TRIzol reagent. The vascular fragments were being lysed in the TRIzol reagent. (B) For sampling myocardial area, laser cut lines were placed not to include microscopically visible vasculatures and infiltrating cells in the area of interest.

Male Wistar-Kyoto rats (WKY) and stroke-prone spontaneously hypertensive rats (SHR-SP) were purchased from SLC (Shizuoka, Japan) and housed under standard conditions of humidity, room temperature, and a 12:12-hour dark-light cycles. They were provided with free access to tap water and chow.

2.1. Animals. At 24 weeks, blood pressure was measured using a tail-cuff sphygmomanometer (MK-2000ST, Muromachi, Tokyo, Japan), as described previously [4]. Thereafter, rats were anesthetized with intraperitoneal ketamine $(50 \mathrm{mg} / \mathrm{kg})$ and xylazine $(10 \mathrm{mg} / \mathrm{kg})$. Percentage of left ventricular fractional shortening was measured using an echocardiography equipped with a $10 \mathrm{MHz}$ transducer (Aloka, Tokyo, Japan) [5-8]. The next day, rats were euthanized with an overdose of pentobarbital $(100 \mathrm{mg} / \mathrm{kg}$, intraperitoneally). After the rats were perfused with icecold saline $\left(4^{\circ} \mathrm{C}\right)$ at $100 \mathrm{mmHg}$, the heart was removed. The left ventricle was snap-frozen in isopentane/dry ice, embedded in OCT compound, and sectioned with cryostat. The cryosections ( $7 \mu \mathrm{m}$ in thickness) were mounted on icecold PEN-slides (Leica Microsystems, Wetzlar, Germany).

2.2. Laser Microdissection Method. Fresh cryosections were fixed in RNase-free-ethyl acetate (acetic acid:ethanol = $1: 19$ ) and stained with $0.05 \%$ toluidine blue dissolved in RNase-free distilled water. The regions of the cardiac muscle and intramyocardial arteries were identified based on microscopic observation and were separately isolated from the section using LMD6000 system (Leica Microsystems). To collect vascular samples, we consistently placed the laser cut line on the outside border of the medial VSMC layer (Figure 1(A)). The isolated fragments were collected in the cap of an Eppendorf tube (Eppendorf Japan, Tokyo, Japan) containing TRIzol reagent (Life Technologies Japan, Tokyo, Japan). Muscular samples were dissected from the myocardium area without microscopically visible vasculatures and infiltrating cells (Figure 1(B)). The vascular sample included 40 crosssections of the intramyocardial arteries for each animal. The muscular fragments with a total of $6 \times 10^{4} \mu \mathrm{m}^{2}$ were collected in each animal.

2.3. Quantitative Real-Time RT-PCR. Total RNA was purified using RNeasy micro (Qiagen, Valencia, CA) according to the manufacturer's instructions. Electropherogram exhibited clear peaks for $18 \mathrm{~S}$ and $28 \mathrm{~S}$ ribosomal RNAs in the purified RNA samples (Figure 2(a)). RNA was reverse transcribed using a High Capacity RNA-to-cDNA kit (GE Health Care, Waukesha, WI). Equal amount of the resulting cDNA was subjected to real-time PCR using the TaqMan Universal PCR Master Mix and a Sequence Detection System model 7700 (Life Technologies Japan) [5, 9, 10]. Primer pairs and TaqMan probes for rat type B-natriuretic peptide (BNP), $\alpha$-smooth muscle actin ( $\alpha$-SMA), and $\beta$-actin were 


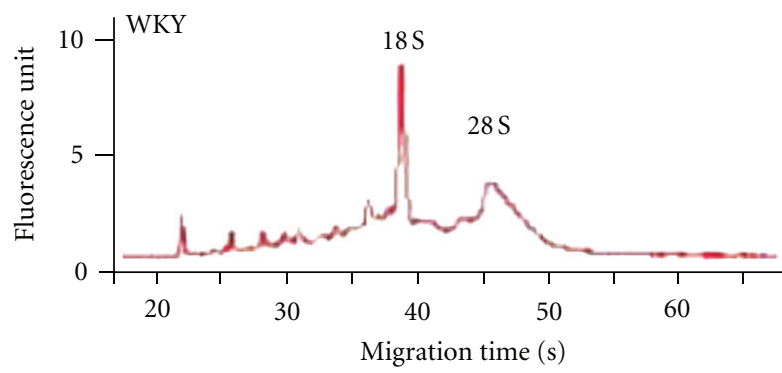

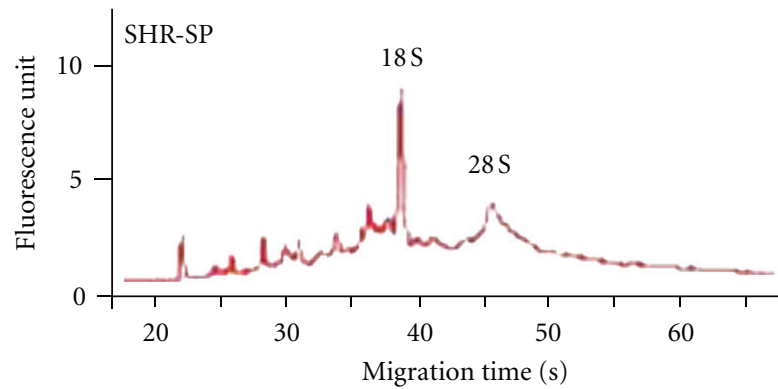

(a)

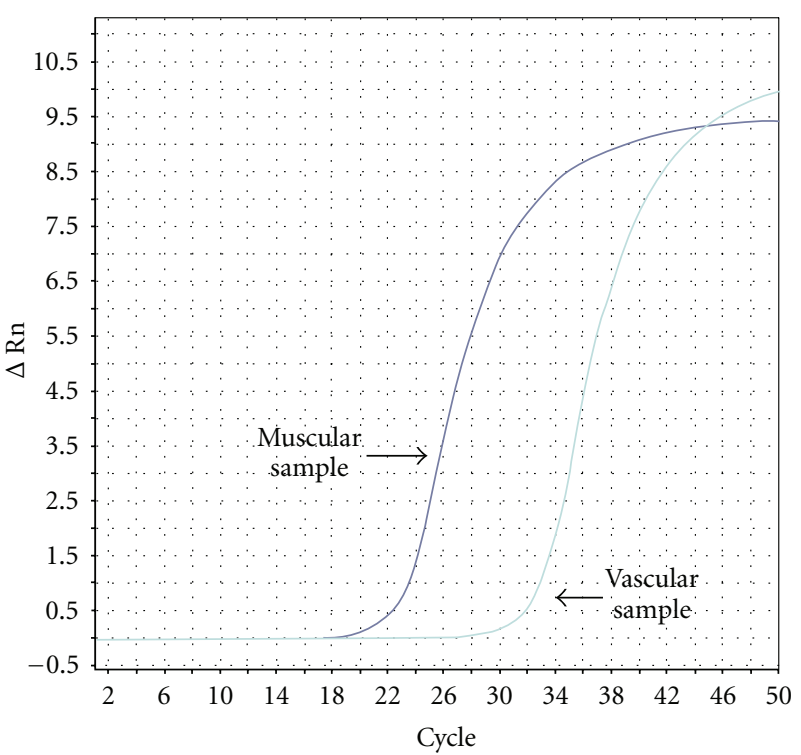

(b)

Figure 2: (a) Representative RNA electrophoregrams of the muscular samples of WKY (top) and SHR-SP (bottom). (b) Representative amplification plots of BNP mRNA in the muscular and vascular samples of WKY.

obtained from GE Health Care. Good amplification plots for target genes were shown in the muscular and vascular samples (Figure 2(b)). Expression level of the target gene was normalized by $\beta$-actin level in each sample.

2.4. Statistical Analysis. Data were expressed as mean \pm SD. Unpaired $t$-test was performed for comparison between two groups.

\section{Results}

At 24 weeks of age, systolic blood pressure was $120.5 \pm$ $12.2 \mathrm{mmHg}$ in WKY $(n=5)$ and $252.5 \pm 16.7 \mathrm{mmHg}$ in SHR-SP $(n=3)(P<0.001)$. Percentage of Left ventricular fractional shortening was $26.8 \pm 4.1 \%$ in WKY $(n=5)$ and $29.8 \pm 5.2 \%$ in SHR-SP $(n=3)$ (no significance). We present one example of real-time RT-PCR analysis in WKY, which showed BNP mRNA expression in the muscular samples, but not in the vascular samples (Figure 3 ). In contrast, $\alpha$-SMA expression was found exclusively in the vascular samples. Next, we compared the expression levels of BNP, a molecular marker of cardiac myocyte hypertrophy, between WKY and SHR-SP (Figure 4). SHR-SP had a significantly greater BNP expression than WKY in the muscular samples. BNP mRNA was not expressed in the vascular samples of WKY and SHRSP.

\section{Discussion}

The present study demonstrated that the LMD method enabled us to selectively collect myocardium and intramyocardial arteries from the heart section. Selective sampling was verified because the expression of cardiac myocytespecific gene marker, BNP, was detected exclusively in the muscular samples, whereas the VSMC-specific marker, $\alpha$ SMA, was expressed only in the vascular samples. Moreover, hypertrophic gene upregulation, as assessed by BNP expression, was significantly greater in the muscular samples obtained from SHR-SP than from WKY.

Recently, it has been reported that the LMD method is useful for selective sampling of the arterial component from the surrounding tissues, for example, the isolation of the arterial lesions in the lung specimen of patients with familiar pulmonary hypertension [11] and the isolation of the collateral vessels in the ischemic hindlimb in mice [12]. Also, the LMD method was used for selective sampling of the intimal plaques in the human atherosclerotic lesions $[13,14]$. However, there have been few studies using the LMD method for gene expression analysis of the heart [15]. Thus, we sought to establish the method to selectively collect muscular and vascular samples from the heart sections using the LMD method.

As shown in Figure 1(A), the intramyocardial arterioles were surrounded by thin loose connective tissue separating from the cardiac muscle tissue. Thus, the microscopic guidance allows us to easily isolate vascular samples. In this study, we successfully collected and analyzed the intramyocardial arteries with a diameter of approximately $50 \mu \mathrm{m}$. This finding was in line with the previous studies demonstrating that relatively small vascular samples, such as intrapulmonary arteries at a size between 50 and $200 \mu \mathrm{m}$ and intrarenal arterioles with a diameter of approximately $100 \mu \mathrm{m}$, were selectively isolated using the LMD method $[16,17]$. In contrast, it was necessary that we consistently took a great care in isolating muscular samples by avoiding 


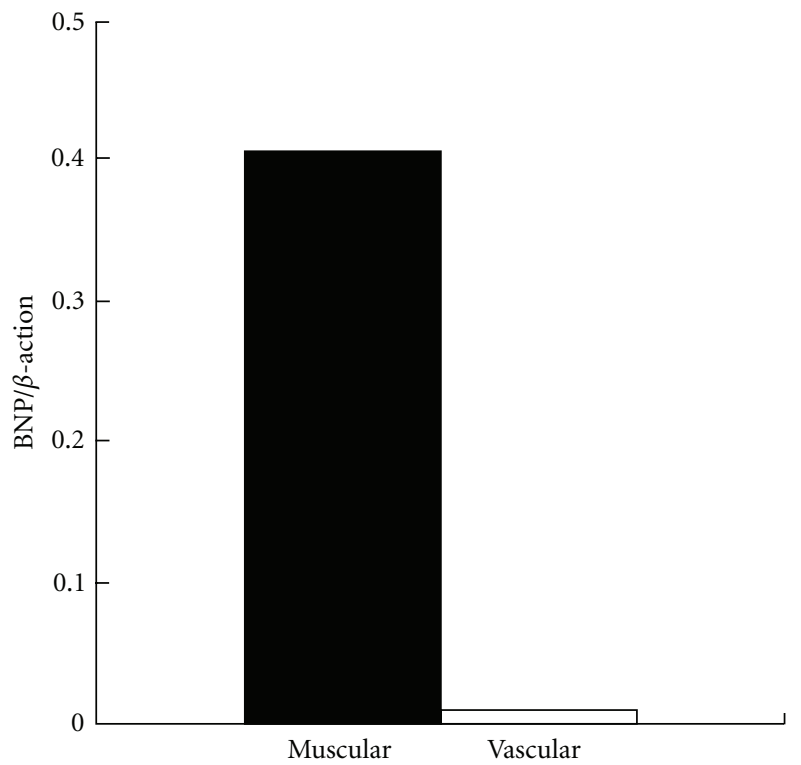

(a)

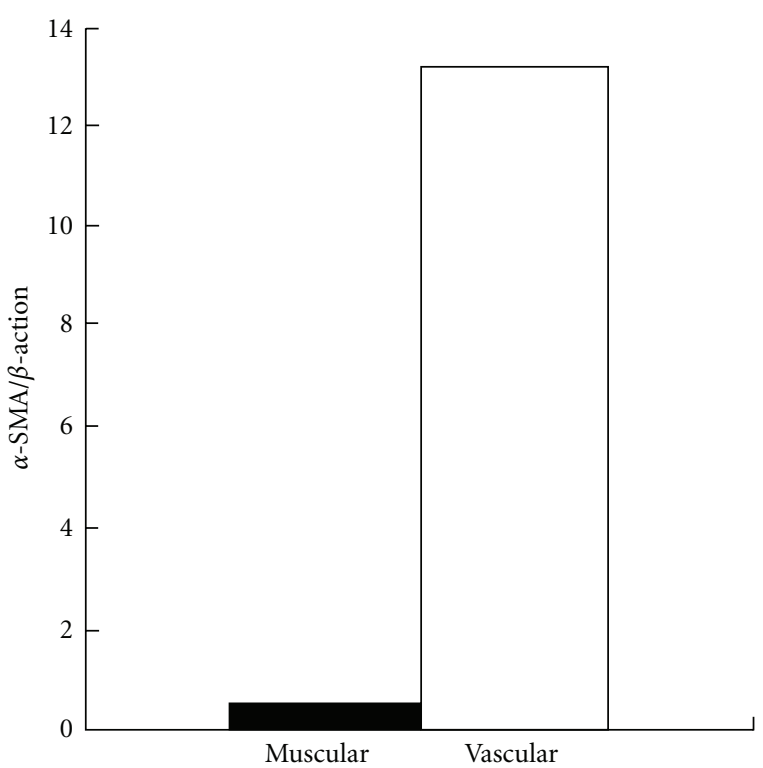

(b)

FiguRE 3: BNP and $\alpha$-SMA mRNA expression in the muscular (closed column) and vascular (open column) samples obtained from the heart of WKY $(n=2)$. Expression level of the target gene was normalized by $\beta$-actin level in each sample.

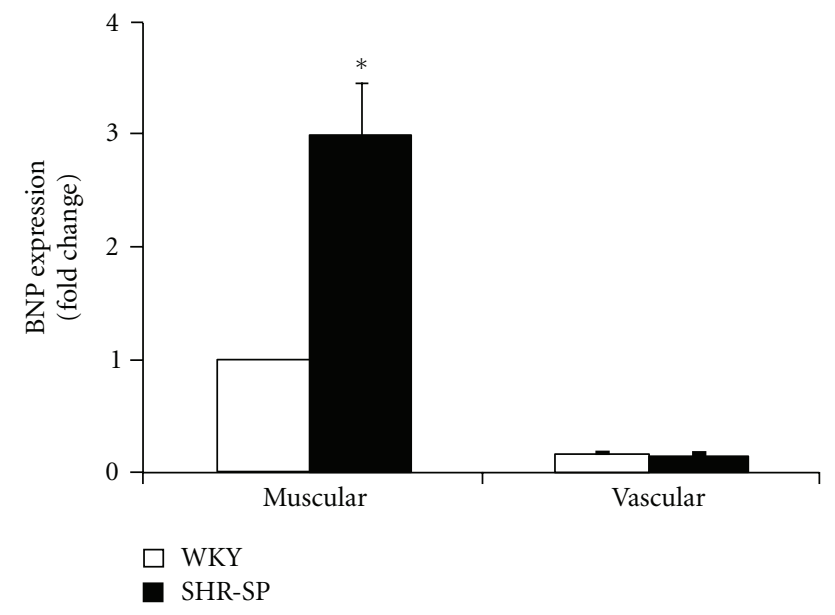

FIGURE 4: BNP mRNA expression in the muscular and vascular samples obtained from the heart of WKY (open column) and SHRSP (closed column). BNP expression levels were expressed as fold change from the muscular sample of WKY. Unpaired $t$-test was used for comparison of initial data before expression as fold changes. Bar $=1 \times \mathrm{SD}(n=3) . * P<0.05$ versus $\mathrm{WKY}$.

microscopically visible vessels and infiltrating cells. The mRNA expressions of BNP and $\alpha$-SMA were not detected in the vascular and muscular samples, respectively (Figure 3 ). Moreover, the BNP mRNA upregulation associated with cardiac hypertrophy was documented specifically in the muscular samples in SHR-SP (Figure 4). These findings suggested that the contamination of cardiac myocytes in the vascular samples or VSMCs in the muscular samples was negligible.
In conclusion, the LMD method enabled us to separately collect the muscular and vascular samples from the myocardial sections and to selectively evaluate the mRNA expression changes in individual tissue component.

\section{Acknowledgments}

This study was supported in part by a grant for the Science Frontier Research Promotion Centers (Cardiovascular Research Institute) and by grants-in-aid for scientific research (A. Ikeda and $\mathrm{H}$. Kai) from the Ministry of Education, Culture, Sports, Science, and Technology Japan. The authors thank Katsue Shiramizu, Kimiko Kimura, Miyuki Nishigata, Miho Kogure, and Makiko Kiyohiro for their skillful technical assistance.

\section{References}

[1] K. T. Weber, C. G. Brilla, and J. S. Janicki, "Myocardial fibrosis: functional significance and regulatory factors," Cardiovascular Research, vol. 27, no. 3, pp. 341-348, 1993.

[2] A. Nicoletti and J. B. Michel, "Cardiac fibrosis and inflammation: interaction with hemodynamic and hormonal factors," Cardiovascular Research, vol. 41, no. 3, pp. 532-543, 1999.

[3] T. Tachikawa and T. Irie, "A new molecular biology approach in morphology: basic method and application of laser microdissection," Medical Electron Microscopy, vol. 37, no. 2, pp. 82-88, 2004.

[4] H. Kajimoto, H. Kai, H. Aoki et al., "Inhibition of eNOS phosphorylation mediates endothelial dysfunction in renal failure: new effect of asymmetric dimethylarginine," Kidney International, vol. 81, no. 8, pp. 762-768, 2012.

[5] F. Kuwahara, H. Kai, K. Tokuda et al., "Transforming growth factor- $\beta$ function blocking prevents myocardial fibrosis and 
diastolic dysfunction in pressure-overloaded rats," Circulation, vol. 106, no. 1, pp. 130-135, 2002.

[6] F. Kuwahara, H. Kai, K. Tokuda et al., "Hypertensive myocardial fibrosis and diastolic dysfunction-another model of inflammation?" Hypertension, vol. 43, no. 4, pp. 739-745, 2004.

[7] T. Mori, H. Kai, H. Kajimoto et al., "Enhanced cardiac inflammation and fibrosis in ovariectomized hypertensive rats: a possible mechanism of diastolic dysfunction in postmenopausal women," Hypertension Research, vol. 34, no. 4, pp. 496-502, 2011.

[8] H. Kudo, H. Kai, H. Kajimoto et al., "Exaggerated blood pressure variability superimposed on hypertension aggravates cardiac remodeling in rats via angiotensin II system-mediated chronic inflammation," Hypertension, vol. 54, no. 4, pp. 832838, 2009.

[9] K. Kusaba, H. Kai, M. Koga et al., "Inhibition of intrinsic interferon- $\gamma$ function prevents neointima formation after balloon injury," Hypertension, vol. 49, no. 4, pp. 909-915, 2007.

[10] M. Koga, H. Kai, H. Yasukawa et al., "Inhibition of progression and stabilization of plaques by postnatal interferon- $\gamma$ function blocking in ApoE-knockout mice," Circulation Research, vol. 101, no. 4, pp. 348-356, 2007.

[11] R. D. Machado, V. James, M. Southwood et al., "Investigation of second genetic hits at the BMPR2 locus as a modulator of disease progression in familial pulmonary arterial hypertension," Circulation, vol. 111, no. 5, pp. 607-613, 2005.

[12] N. van Royen, M. Voskuil, I. Hoefer et al., "CD44 regulates arteriogenesis in mice and is differentially expressed in patients with poor and good collateralization," Circulation, vol. 109, no. 13, pp. 1647-1652, 2004.

[13] T. T. Tuomisto, A. Korkeela, J. Rutanen et al., "Gene expression in macrophage-rich inflammatory cell infiltrates in human atherosclerotic lesions as studied by laser microdissection and DNA array: overexpression of HMG-CoA reductase, colony stimulating factor receptors, CD11A/CD18 integrins, and interleukin receptors," Arteriosclerosis, Thrombosis, and Vascular Biology, vol. 23, no. 12, pp. 2235-2240, 2003.

[14] K. Stolle, B. Weitkamp, J. Rauterberg, S. Lorkowski, and P. Cullen, "Laser microdissection-based analysis of mRNA expression in human coronary arteries with intimal thickening," Journal of Histochemistry and Cytochemistry, vol. 52, no. 11, pp. 1511-1518, 2004.

[15] S. Moniotte, J. L. Vaerman, M. M. Kockx et al., "Real-time RT-PCR for the detection of beta-adrenoceptor messenger RNAs in small human endomyocardial biopsies," Journal of Molecular and Cellular Cardiology, vol. 33, no. 12, pp. 21212133, 2001.

[16] R. T. Schermuly, S. S. Pullamsetti, G. Kwapiszewska et al., "Phosphodiesterase 1 upregulation in pulmonary arterial hypertension: target for reverse-remodeling therapy," Circulation, vol. 115, no. 17, pp. 2331-2339, 2007.

[17] M. J. Soler, M. Ye, J. Wysocki, J. William, J. Lloveras, and D. Batlle, "Localization of ACE2 in the renal vasculature: amplification by angiotensin II type 1 receptor blockade using telmisartan," The American Journal of Physiology, vol. 296, no. 2, pp. F398-F405, 2009. 


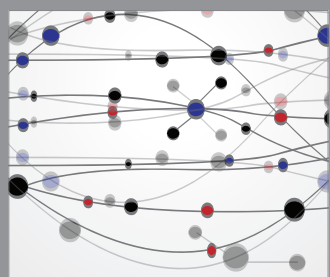

The Scientific World Journal
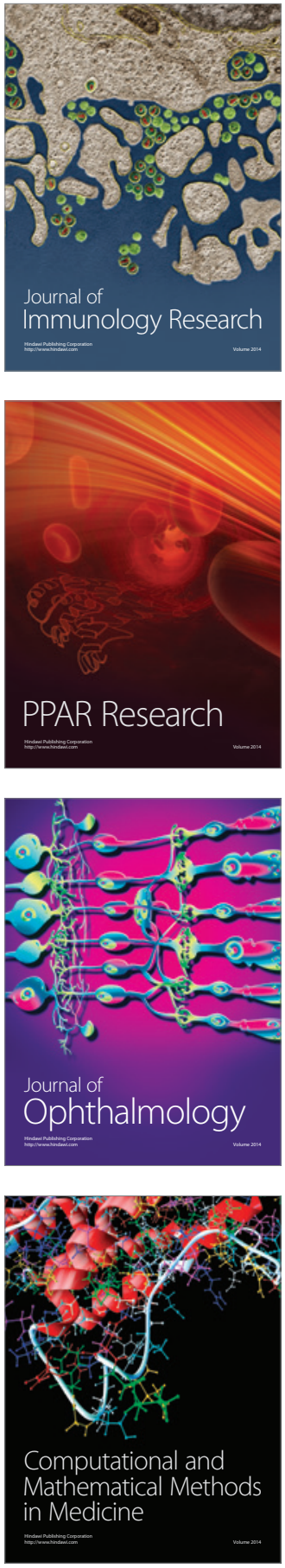

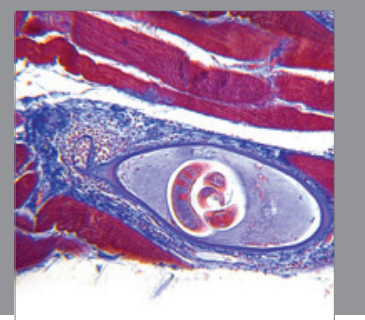

Gastroenterology

Research and Practice
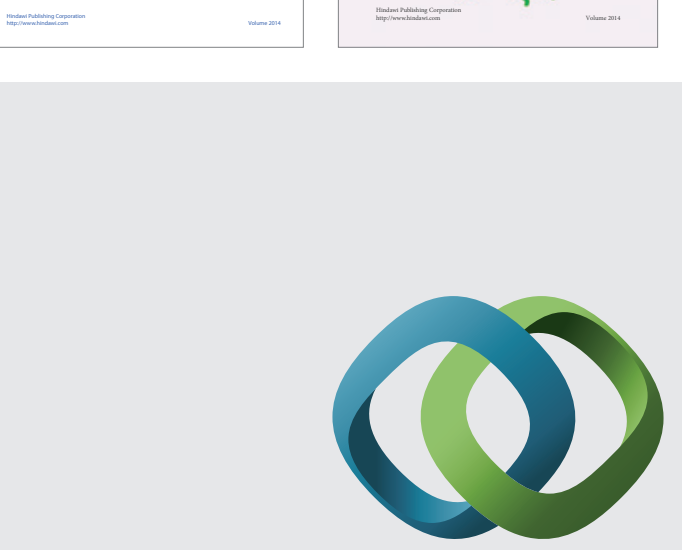

\section{Hindawi}

Submit your manuscripts at

http://www.hindawi.com
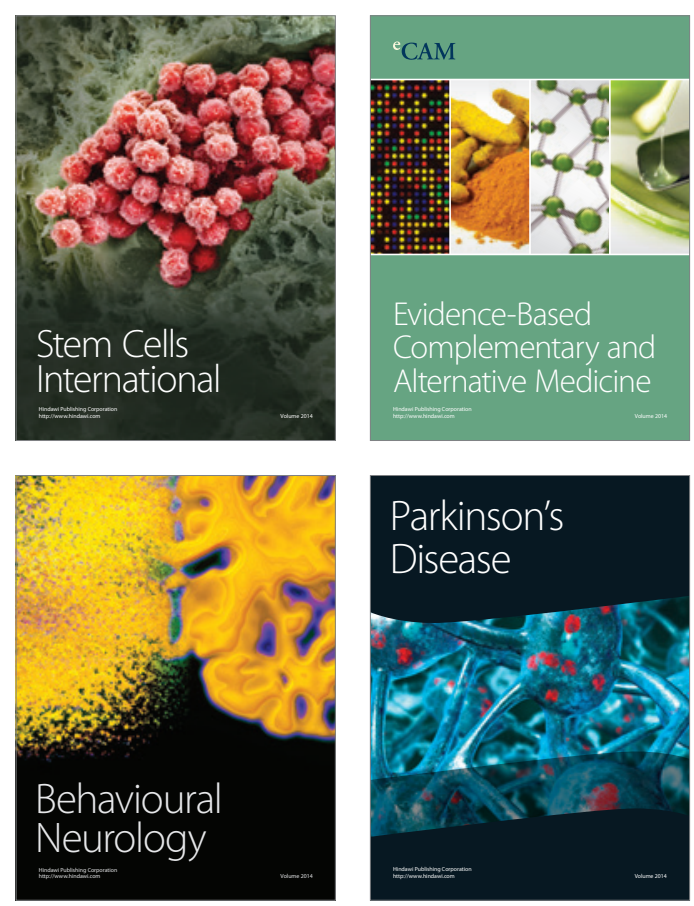

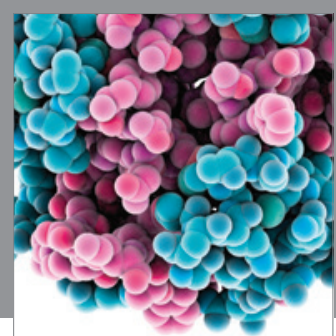

Journal of
Diabetes Research

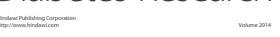

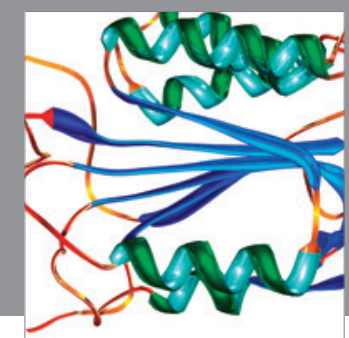

Disease Markers
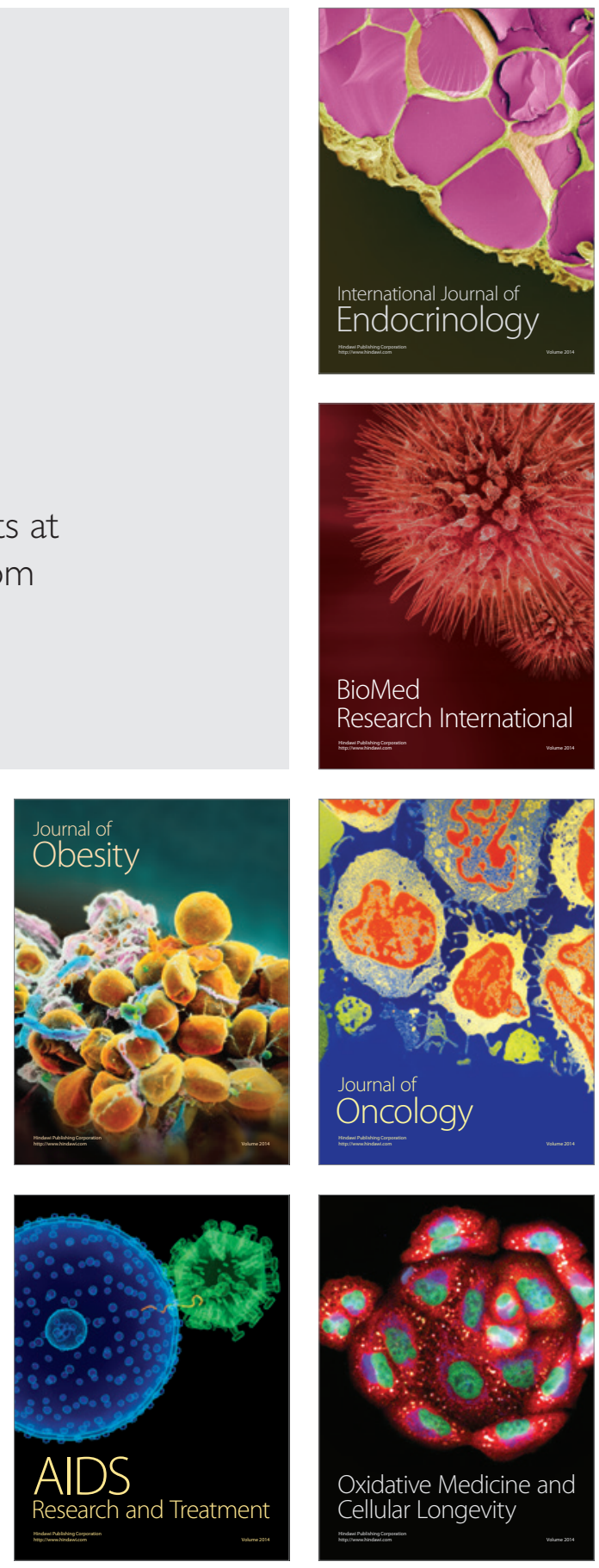